\title{
Precision Structural Engineering of Self-Rolled-up 3D Nanomembranes Guided by Transient Quasi-Static FEM Modeling
}

\author{
Wen Huang, ${ }^{\dagger, \ddagger}$ Seid Koric, ${ }^{\S, \|}$ Xin Yu, ${ }^{\ddagger}$ K. Jimmy Hsia, ${ }^{\S, \ddagger}$ and Xiuling Li ${ }^{*, \dagger, \ddagger}$ \\ ${ }^{\dagger}$ Department of Electrical and Computer Engineering, ${ }^{\ddagger}$ Micro and Nanotechnology Laboratory, ${ }^{\S}$ Department of Mechanical Science \\ and Engineering, and "National Center for Supercomputing Applications, University of Illinois at Urbana-Champaign, Urbana, \\ Illinois 61801, United States
}

\section{Supporting Information}

ABSTRACT: Micro- and nanoscale tubular structures can be formed by straininduced self-rolled-up nanomembranes. Precision engineering of the shape and dimension determines the performance of devices based on this platform for electronic, optical, and biological applications. A transient quasi-static finite element method (FEM) with moving boundary conditions is proposed as a general approach to design diverse types of three-dimensional (3D) rolled-up geometries. This method captures the dynamic release process of membranes through etching driven by mismatch strain and accurately predicts the final dimensions of rolled-up structures. Guided by the FEM modeling, experimental demonstration using silicon nitride membranes was achieved with unprecedented precision including controlling fractional turns of a rolled-up membrane, anisotropic rolling to form helical structures, and local stress control for 3D hierarchical architectures.

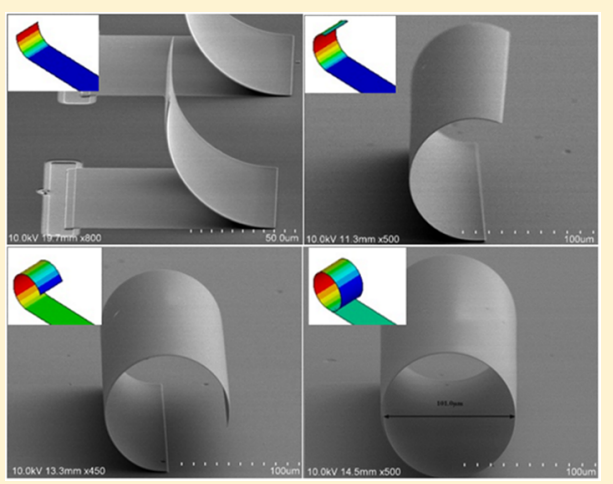

KEYWORDS: Geometry engineering, self-rolled-up nanomembrane tube, transient quasi-static, finite element method

$\mathrm{T}$ he technology to produce strain-induced self-rolled-up nanomembrane tubes has attracted much attention in the nanofabrication research community since the pioneer work by Prinz et al. in 2000. ${ }^{1}$ These tubes have been proposed as a new platform for the design of novel devices for electronics, photonic, and biological applications with unprecedented functionalities and extremely small footprint. ${ }^{2-8}$ The essential element of the technology involves planar, multilayered, patterned surface structures containing one or several strained layers supported by a sacrificial layer. Nanomembrane deposition methods include plasma enhanced chemical vapor deposition (PECVD), metal-organic chemical vapor deposition (MOCVD), molecular beam epitaxy (MBE), and so forth. The tube material systems can usually be classified into four main categories: amorphous films, ${ }^{9-12}$ epitaxial single crystal films, ${ }^{1,4,13-15}$ strained polymer bilayers, ${ }^{16}$ and hybrid material systems. $^{9,16-21}$ Various material systems, such as epitaxially deposited lattice-matched heterojuctions, ${ }^{1,4,13-15}$ spun-on layers, ${ }^{10,11}$ and semiconductor substrates, ${ }^{20,22,23}$ have been used as the sacrificial layers.

Curving of multilayered thin film structures due to mismatch strain between layers is well understood and documented by Freund and Suresh. ${ }^{24}$ When the mismatch strain is sufficiently large to bring the layered structures into the range of geometrically nonlinear response, bifurcation occurs that leads to a rolled-up tubular shape. ${ }^{24,25}$ In thin film roll-up experiments, the mismatch strain driven buckling in layered structures is activated by releasing the constraints of the substrate via etching off the sacrificial layer. The rolling process continues until the entire sacrificial layer underneath the layered thin film is etched away, resulting in a multiple-turn three-dimensional (3D) tubular structure on the substrate. The diameter of the innermost tube is thus a key geometrical parameter determining the final dimension. For devices where performance is sensitive to geometric shapes, such as high frequency electronics and optics, it is crucial to precisely control the inner diameter.

Analytical and numerical methods have been developed to quantitatively calculate or simulate the value of the inner diameter for tubes with different layered structures from macroscopic level down to nanoscale level. As early as 1925, a model for bimetallic thermostat deflection was developed by Timoshenko. $^{26}$ This classic continuum model for macroscopic structures has since been modified to take into consideration the surface stress to give a more accurate calculation for the bending curvature of strained bilayer nanoscale thin films. ${ }^{27,28}$ The finite element method (FEM) has also been applied to study the effect of geometric dimension on the deformation of a bilayer structure under static conditions. ${ }^{29}$ Both the analytical and numerical methods have been successfully used to accurately estimate the inner diameter for many specific layered structures. $^{30,31}$ However, in modern electronics and optics applications, thin film structures often contain more than two sublayers, and the top strained layer is usually patterned to

Received: July 11, 2014

Revised: October 7, 2014 
perform specific functions. The patterned layer makes the mismatch strain (and thus stresses) distributions across the entire strained layer nonuniform. The complexity of the practical topography makes it impossible to use an analytical method to determine the inner diameter of the final rolled-up structure. Numerical method is preferred in these circumstances. Moreover, to understand the kinetics involved in the rolling process dynamic simulations must be carried out.

In this paper, a method of using FEM to do transient quasidynamic simulations of the rolling up process of any strained layer construction is employed. Extremely high accuracy of the numerical modeling method is confirmed by comparing the numerical and the experimental results. Examples of complex rolled-up 3D hierarchical structures are also demonstrated by utilizing the local stress variation introduced by prepatterned straining layer.

A silicon nitride $\left(\mathrm{SiN}_{x}\right)$ bilayer structure is studied here as a model system. The bilayer structure consists of a bottom low frequency (LF) $\mathrm{SiN}_{x}$ layer (under compressive stress before releasing from constraint) and the top high frequency (HF) $\mathrm{SiN}_{x}$ layer (under tensile stress before releasing from constraint), as shown in Figure 1. Initially a fixed boundary condition is applied to all nodes at the bottom of $\mathrm{LF} \mathrm{SiN}_{x}$ layer to model the effect of the sacrificial layer. The materials are assumed to be isotropic and linear elastic since the proposed FEM modeling stays in elastic region but modeled with geometric nonlinearities due to large deformation. Shell element is used to model multiple-layer structures, and its accuracy in modeling composite shells is governed by the Mindlin-Reissner shell theory. Different thicknesses and material properties are assigned to each layer. The Young's modulus $E$ for both PECVD LF $\mathrm{SiN}_{x}$ and HF $\mathrm{SiN}_{x}$ thin films with similar growth condition was reported to be $210 \mathrm{GPa}^{32-34}$ Poisson coefficient was chosen to be 0.28 for both $\mathrm{LF} \mathrm{SiN}_{x}$ and HF $\mathrm{SiN}_{x}$ thin films in simulation. Residual stresses of both LF $\mathrm{SiN}_{x}$ and $\mathrm{HF} \mathrm{SiN}_{x}$ are modeled by a fictitious thermal expansion in FEM. The same temperature increment is assigned to the nodes of all shell elements. To simulate the compressive and tensile stresses, different coefficients of thermal expansion were assigned to the $\mathrm{LF} \mathrm{SiN}_{x}$ and $\mathrm{HF}$ $\mathrm{SiN}_{x}$ layers. The premeasured residual stresses of each layer by a FSM 500TC metrology tool can then be induced by applying a proper temperature increment such that the measured residual stresses for each thin film layer are achieved. Thermal coefficient of $\mathrm{LF} \mathrm{SiN}_{x}$ is taken from literature. The fictitious temperature increment of $\mathrm{LF} \mathrm{SiNx}$ is then determined to be $1450{ }^{\circ} \mathrm{C}$ to achieve the measured value of residual stress in LF $\mathrm{SiN}_{x}$. For other materials listed in Table 2, their thermal coefficients are fitted for each material to reach its respective measured residual stress level when the fictitious temperature increment is fixed at $1450{ }^{\circ} \mathrm{C}$.

A moving boundary condition is used to model the etching of the sacrificial layer. The rolling process of the strained membrane is a nonlinear large deformation transient quasidynamic process. This process is simulated by a series of FEM simulations of static deformation by releasing the constraints on the bottom segments in sequence. In our simulations, the length of each segment is set to be less than $1 / 200$ of the circumference of the first turn. To apply the moving boundary condition, a simulation loop shown in Figure 1 is realized. The loop starts from Figure la by applying a fixed boundary condition at the bottom of the bilayer to model the sacrificial layer. In the next step, shown in Figure 1b, the constraint on

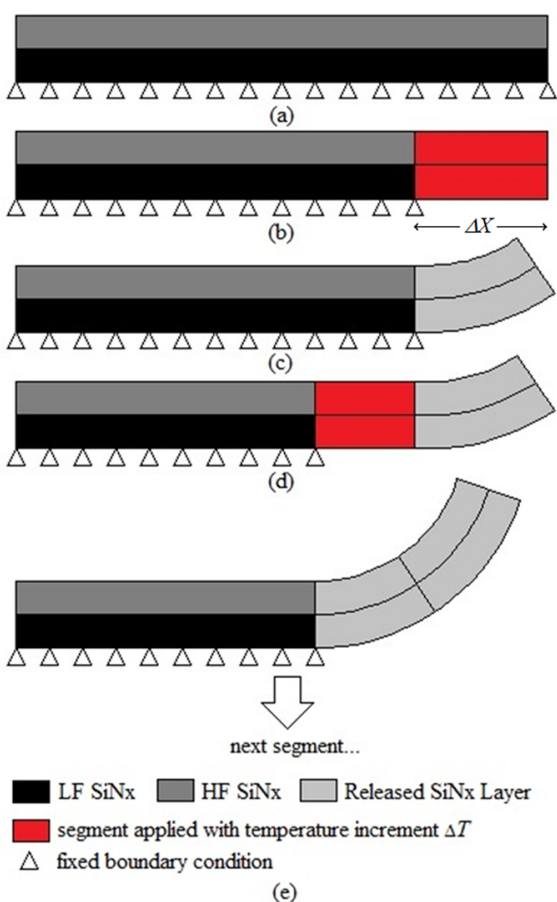

Figure 1. Transient quasi-static FEM modeling of the rolling process of bilayer membrane. (a) Initial structure with applied fixed boundary condition to all element nodes. (b) Unfix the first segment and apply a temperature increment $\Delta T$ to it. (c) Update the structure after static simulation. (d) Unfix the next segment and apply a temperature increment $\Delta T$ to it. (e) Update the structure after static simulation and continue the loop until the last segment is simulated.

Table 1. Comparison of the Inner Diameter Experiment Data with the Simulation Results

\begin{tabular}{ccccc} 
sample \# & $\begin{array}{c}\text { thickness of } \\
\mathrm{LF} \mathrm{SiN}_{x} \\
(\mathrm{~nm})\end{array}$ & $\begin{array}{c}\text { thickness of HF } \\
\mathrm{SiN}_{x} / \mathrm{Ni} / \mathrm{Au} \\
(\mathrm{nm})\end{array}$ & $\begin{array}{c}\text { measured } \\
\text { inner diameter } \\
(\mu \mathrm{m})\end{array}$ & $\begin{array}{c}\text { calculated } \\
\text { inner diameter } \\
(\mu \mathrm{m})\end{array}$ \\
1 & 16 & $20 / 0 / 0$ & 5.1 & 5.2 \\
2 & 31 & $40 / 0 / 0$ & 10.8 & 10.2 \\
3 & 39 & $50 / 0 / 0$ & 12.3 & 12.7 \\
4 & 62 & $80 / 0 / 0$ & 19.5 & 20.4 \\
5 & 93 & $120 / 0 / 0$ & 29.9 & 30.5 \\
6 & 40 & $30 / 5 / 30$ & 13.8 & 13.5 \\
7 & 155 & $200 / 0 / 0$ & 49.8 & 50.8 \\
8 & 300 & $400 / 0 / 0$ & 101.0 & 100.3 \\
\hline
\end{tabular}

Table 2. Material Properties Set in FEM Simulation ${ }^{a}$

$\begin{array}{lccccc} & \begin{array}{c}\text { residual } \\ \text { stress } \\ (\mathrm{MPa})\end{array} & \begin{array}{c}\text { Young's } \\ \text { modulus } \\ (\mathrm{GPa})\end{array} & \begin{array}{c}\text { Poisson } \\ \text { coefficient }\end{array} & \begin{array}{c}\text { thermal } \\ \text { expansion } \\ \text { coefficient } \\ \left(1 /{ }^{\circ} \mathrm{C}\right)\end{array} & \begin{array}{c}\text { temperature } \\ \text { increment } \\ \left({ }^{\circ} \mathrm{C}\right)\end{array} \\ \text { sublayer } & -1133 & 210 & 0.28 & 2.75 \times 10^{-6} & 1450 \\ \mathrm{LF} \mathrm{SiN}_{x} & +387 & 210 & 0.28 & -9.61 \times 10^{-7} & 1450 \\ \mathrm{HF} \mathrm{SiN}_{x} & +790 & 200 & 0.31 & -1.9 \times 10^{-6} & 1450 \\ \mathrm{Ni} & +380 & 79 & 0.44 & -1.85 \times 10^{-6} & 1450 \\ \mathrm{Au} & +790\end{array}$

${ }^{a}$ Signs - and + for the residual stress denote compressive and tensile stresses, respectively.

the first segment $\Delta X$ is released and a fictitious temperature increment $\Delta T=1450{ }^{\circ} \mathrm{C}$ is applied to all nodes associated with this segment. After that, static simulation is performed to obtain an updated geometry shown in Figure 1c. By repeating the loop, the next segment is released and the same temperature 

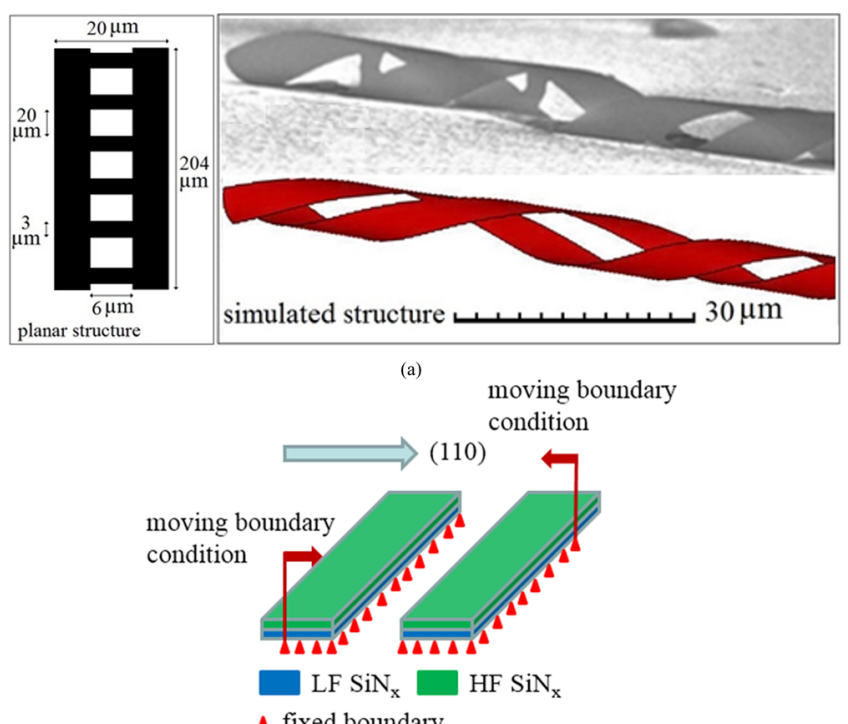

- fixed boundary

Figure 2. A double helix structure rolled-up by anisotropic release. (a) Geometry comparison between the fabricated structure and the simulated structure. (b) Methodology to model anisotropic etching of the single crystal silicon sacrificial layer.

increment $\Delta T$ is applied to obtain the next updated geometry as shown in Figure 1d. The loop repeats until the last segment is released.
Supporting Information Figure S1 shows different stages of the rolling process from the transient quasi-static simulation of a $\mathrm{SiN}_{x}$ bilayer structure. Different colors in the strained membrane represent the amount of displacement. The transient quasi-static rolling process can be illustrated by combining a series of simulation results in a time sequence (movies are provided in the Supporting Information). Table 1 compares the values of the measured inner diameter of several $\mathrm{SiN}_{x}$ tube samples with the corresponding designed inner diameters, showing a high accuracy of the method. Samples \#7 and \#8 show the rolled-up $\mathrm{SiN}_{x}$ bilayer with the largest inner diameters (thickest film) reported so far, which was achieved under the optimized fabrication process preventing $\mathrm{SiN}_{x}$ from self-cracking before rolling-up (more information can be found in the Supporting Information). All samples have $20 \mathrm{~nm}$ thick $\mathrm{Ge}$ as the sacrificial layer. Sample \#6 has $5 \mathrm{~nm} \mathrm{Ni} / 30 \mathrm{~nm} \mathrm{Au}$ deposited on top of the bilayer, whereas all other samples are of simple $\mathrm{SiN}_{x}$ bilayer structures. SEM pictures with marked inner diameter of the samples can be found in Figure S2 in the Supporting Information. Table 2 summarizes the material properties used in the simulations. For cases where there may be more than two strained layer, simulations were carried out in a similar way as described above.

Other complex rolled-up structures can be fabricated by taking advantage of anisotropic etching of the sacrificial layer. $^{34,35}$ Complicated three-dimensional structures, such as spirals, are difficult to simulate by traditional numerical methods. However, such processes can be simulated by controlling the moving boundary conditions to mimic the

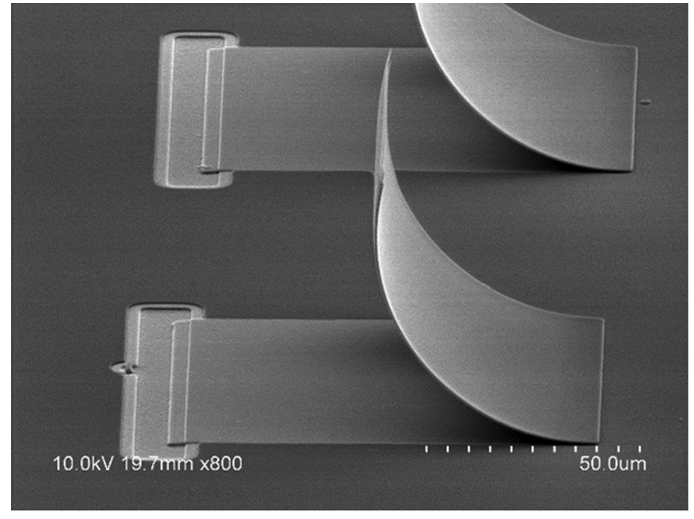

(a)

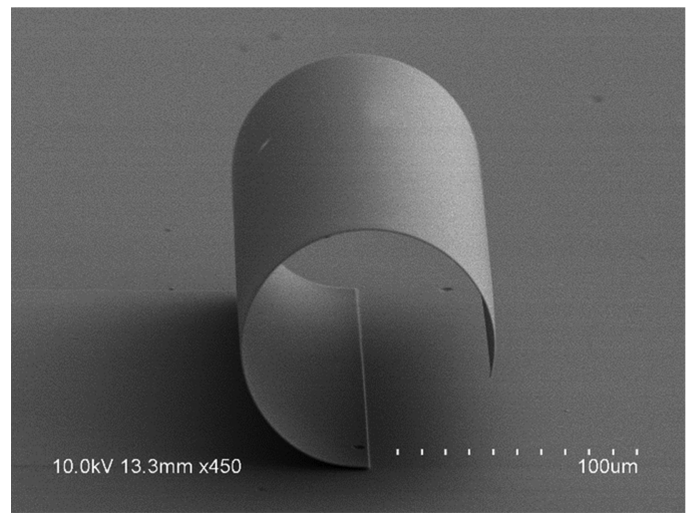

(c)

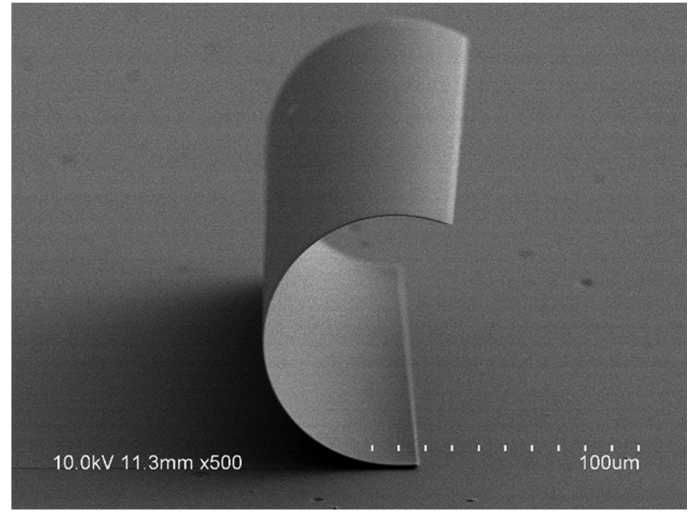

(b)

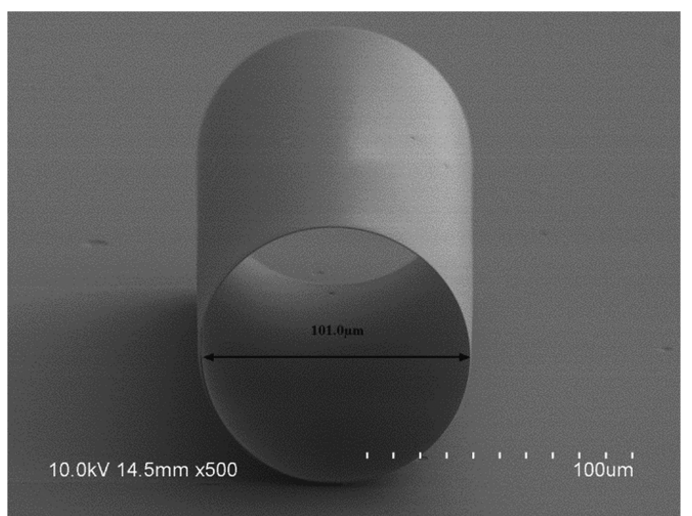

(d)

Figure 3. SEM images of precisely fabricated $100 \mu \mathrm{m}$ inner diameter rolled-up $\mathrm{SiN}_{x}$ bilayer structures with designed fraction of turns. (a) One-fourth turn. (b) Two-fourths turn. (c) Three-fourths turn. (d) Full turn with inner diameter measured as indicated. 


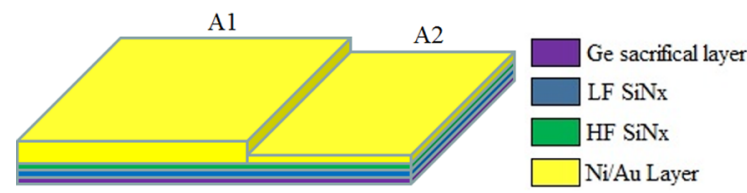

(a)

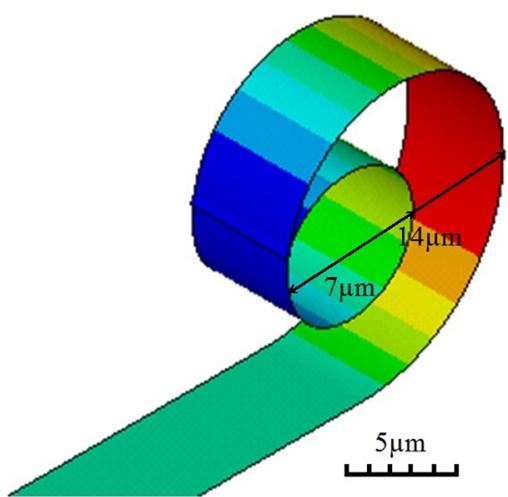

(b)

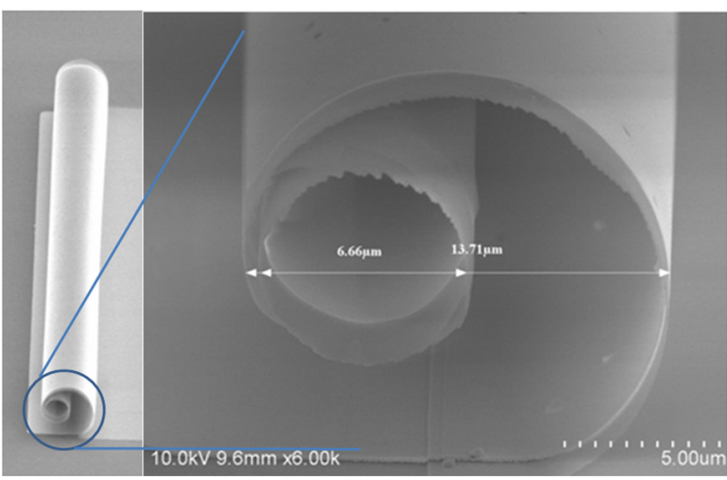

(c)

Figure 4. A coaxial structure rolled-up by local stress control. (a) Design of the planar structure required to form a coaxial rolled-up structure with two different inner diameters. (b) Simulated rolled up structure with inner diameters 7 and $14 \mu \mathrm{m}$ for the first turn and the second turn. (c) Fabricated structure with measured inner diameter. The inner diameter is $6.66 \mu \mathrm{m}$ for the first turn and $13.71 \mu \mathrm{m}$ for the second turn. The thickness of LF $\mathrm{SiN}_{x}$ is $20 \mathrm{~nm}$ and the thickness of $\mathrm{HF} \mathrm{SiN}_{x}$ is $20 \mathrm{~nm}$. The thickness of metal layer in Area A1 is $5 \mathrm{~nm} \mathrm{Ni} /$ $20 \mathrm{~nm} \mathrm{Au}$. The thickness of metal layer in Area A2 is $5 \mathrm{~nm} \mathrm{Ni} / 100 \mathrm{~nm}$ $\mathrm{Au}$. The thickness of Ge sacrificial layer is $10 \mathrm{~nm}$.

actual anisotropic etching process. As an example, Figure 2a shows the design of ladder-shaped rectangular strip of a $\mathrm{SiN}_{x}$ membrane with dimensions shown in the top-left picture. ${ }^{34}$ It is placed on top of single crystal silicon sacrificial layer oriented at an angle of $15^{\circ}$ clockwise relative to the (110) facets. Anisotropic etching of the sacrificial layer leads to etching of the bottom left and top right corners first and finally results in a left-handed double helix structure. In the FEM modeling, the anisotropic etching process can be represented by moving the fixed boundary conductions along the direction of etching of the sacrificial layer as shown in Figure $2 b$, that is, the front of the fixed boundary in the simulation lies in the direction $15^{\circ}$ relative to the (110) facets. The simulation follows the same loop illustrated above. Because the ladder rungs have the same curvature as the rest of the membrane and play a minor role in the shape change, they are not considered in the current FEM model. The simulation results are illustrated in a movie in the Supporting Information. The simulated rolled up structure shows good agreement with the measured results shown in Figure $2 \mathrm{a}$ on the right.

The FEM modeling allows us to precisely control the number of turns of a rolled-up tube by predesigning the length of the layered structures, which is very important in many applications. Figure 3 shows the scanning electron microscopy (SEM) images of computationally designed $\mathrm{SiN}_{x}$ bilayer rolledup with $1 / 4,2 / 4,3 / 4$, or a full-turn with a $101 \mu \mathrm{m}$ inner diameter. The seamless full-turn tube fabricated shown in Figure $3 \mathrm{~d}$ demonstrates the extremely high accuracy of the FEM model.

In certain applications, local stresses can be engineered by patterning the top strained sublayer, which is used to obtain special rolled-up geometry for electrical, optical and biological functions. More complex 3D shapes may be achieved once the mechanics of rolling deformation is understood and numerical simulations are employed. A simple practical case is shown in Figure $4 \mathrm{a}, \mathrm{Ni} / \mathrm{Au}$ layer is deposited on top of $\mathrm{SiN}_{x}$ bilayer with different thicknesses in two areas A1 and A2. Because FEM allows setting the material properties for different shell elements such as the number of sublayers and sublayer dimensions, it is easy to model this complicated case by defining local area A1 and A2 individually and "gluing" them together to perform transient quasi-static simulations. The rolled-up structure is designed to form a tube-in-tube structure with different inner diameters. With different thicknesses of the metal layer deposited on top, local stress is tunable to form different inner diameters. The inner diameter for the outer tube is designed to double that of the inner tube. Figure $4 \mathrm{~b}$ shows the simulated structure with measured inner diameters of the first and second turn, and Figure $4 \mathrm{c}$ shows the measured inner diameters of the inner and the outer tubes which precisely fit the simulation.

In conclusion, we employed an FEM approach with a moving boundary condition to precisely model the geometry and dimensions of rolled-up structures. The precision of such approach is demonstrated by the excellent agreement between experiments on $\mathrm{SiN}_{x}$ bilayer structure and numerical simulation results. The inner diameters and the number of turns of hollow cylindrical spiral structures can be precisely simulated. Complex structures formed by anisotropic sacrificial layer etching or through local stress control can also be precisely modeled. The universality of this approach has significant impact on the precise control of tubular and curved nanostructures for improving the design accuracy and research efficiency in nanoelectronics, nanophotonics, and biomedical applications.

\section{ASSOCIATED CONTENT}

\section{S Supporting Information}

Detail of experimental and additional figures depicting experiment results. This material is available free of charge via the Internet at http://pubs.acs.org.

\section{AUTHOR INFORMATION}

\section{Corresponding Author}

*E-mail: xiuling@illinois.edu. Phone: +1 (217)265-6354. Fax: +1(217)244-6375.

\section{Author Contributions}

W.H. carried out the simulation and experiment with guidance and supervision from X.L., K.J.H., and S.K. X.Y. contributed to the initial stage of fabrication work. The manuscript was written 
through contributions of all authors. All authors have given approval to the final version of the manuscript.

\section{Funding}

The authors gratefully acknowledge financial support provided by the U.S. Department of Energy, Office of Basic Energy Sciences, Division of Materials Sciences and Engineering under Award No. DE-FG02-07ER46471.

\section{Notes}

The authors declare no competing financial interest.

\section{REFERENCES}

(1) Prinz, V. Y.; Seleznev, V.; Gutakovsky, A.; Chehovskiy, A.; Preobrazhenskii, V.; Putyato, M.; Gavrilova, T. Phys. E 2000, 6 (1), 828-831.

(2) Huang, W.; Yu, X.; Comberiate, T.; Qiu, C.-W.; Schutt-Aine, J. E.; Li, X. In Miniaturized on-chip passive devices based on self-rolled-up SiN $x$ nanomembrane inductive tube; 71st Annual Device Research Conference (DRC); IEEE: Bellingham, WA, 2013; pp 227-228.

(3) Huang, W.; Yu, X.; Froeter, P.; Xu, R.; Ferreira, P.; Li, X. Nano Lett. 2012, 12 (12), 6283-6288.

(4) Li, X. J. Phys. D: Appl. Phys. 2008, 41 (19), 193001.

(5) Li, X. Adv. Opt. Photonics 2011, 3 (4), 366-387.

(6) Tian, Z.; Veerasubramanian, V.; Bianucci, P.; Mukherjee, S.; Mi, Z.; Kirk, A. G.; Plant, D. V. Opt. Express 2011, 19 (13), 12164-12171.

(7) Yu, M.; Huang, Y.; Ballweg, J.; Shin, H.; Huang, M.; Savage, D. E.; Lagally, M. G.; Dent, E. W.; Blick, R. H.; Williams, J. C. ACS Nano 2011, 5 (4), 2447-2457.

(8) Froeter, P.; Huang, Y.; Cangellaris, O. V.; Huang, W.; Dent, E. W.; Gillette, M. U.; Williams, J. C.; Li, X. ACS Nano 2014; DOI: $10.1021 / \mathrm{nn} 504876 \mathrm{y}$.

(9) Arora, W. J.; Nichol, A. J.; Smith, H. I.; Barbastathis, G. Appl. Phys. Lett. 2006, 88 (5), 053108.

(10) Huang, G.; Kiravittaya, S.; Quiñones, V. B.; Ding, F.; Benyoucef, M.; Rastelli, A.; Mei, Y.; Schmidt, O. Appl. Phys. Lett. 2009, 94 (14), 141901.

(11) Mei, Y.; Huang, G.; Solovev, A. A.; Urena, E. B.; Mönch, I.; Ding, F.; Reindl, T.; Fu, R. K.; Chu, P. K.; Schmidt, O. G. Adv. Mater. 2008, 20 (21), 4085-4090.

(12) Schulze, S.; Huang, G.; Krause, M.; Aubyn, D.; Quiñones, V. A. B.; Schmidt, C. K.; Mei, Y.; Schmidt, O. G. Adv. Eng. Mater. 2010, 12 (9), B558-B564.

(13) Mi, Z.; Bianucci, P.; Dastjerdi, M. H.; Mukherjee, S.; Tian, Z.; Veerasubramanian, V.; Kirk, A. G.; Plant, D. V. In 1.3-1.55 $\mu \mathrm{m}$ SelfOrganized InAs Quantum Dot Tube Nanoscale Lasers on Silicon; 2011 IEEE Photonics Conference (PHO); IEEE: Bellingham, WA, 2011; pp 535-536.

(14) Chun, I.; Verma, V.; Elarde, V.; Kim, S.; Zuo, J.; Coleman, J.; Li, X. J. Cryst. Growth 2008, 310 (7), 2353-2358.

(15) Chun, I. S.; Li, X. IEEE Trans. Nanotechnol. 2008, 7 (4), 493495.

(16) Luchnikov, V.; Kumar, K.; Stamm, M. J. Micromechan. Microeng. 2008, 18 (3), 035041.

(17) Songmuang, R.; Rastelli, A.; Mendach, S.; Deneke, C.; Schmidt, O. Microelectron. Eng. 2007, 84 (5), 1427-1430.

(18) Golod, S.; Prinz, V. Y.; Wagli, P.; Zhang, L.; Kirfel, O.; Deckhardt, E.; Glaus, F.; David, C.; Grutzmacher, D. Appl. Phys. Lett. 2004, 84 (17), 3391-3393.

(19) Luo, J.; Huang, R.; He, J.; Fu, Y.; Flewitt, A.; Spearing, S.; Fleck, N.; Milne, W. Sens. Actuators A 2006, 132 (1), 346-353.

(20) Dai, L.; Zhang, L. Nanoscale 2013, 5 (3), 971-976.

(21) Moiseeva, E.; Senousy, Y.; McNamara, S.; Harnett, C. J. Micromechan. Microeng. 2007, 17 (9), N63.

(22) Seleznev, V.; Prinz, V. Y.; Aniskin, V.; Maslov, A. J. Appl. Mech. Tech. Phys. 2009, 50 (2), 291-296.

(23) Golod, S.; Prinz, V. Y.; Mashanov, V.; Gutakovsky, A. Semicond. Sci. Technol. 2001, 16 (3), 181.
(24) Freund, L. B.; Suresh, S. Thin film materials: stress, defect formation and surface evolution; Cambridge University Press: New York, 2004.

(25) Scott, S. A.; Lagally, M. G. J. Phys. D: Appl. Phys. 2007, 40 (4), R75.

(26) Timoshenko, S. J. Opt. Soc. Am. 1925, 11 (3), 233-255.

(27) Zang, J.; Liu, F. Appl. Phys. Lett. 2008, 92 (2), 021905.

(28) Zang, J.; Huang, M.; Liu, F. Phys. Rev. Lett. 2007, 98 (14), 146102.

(29) Chun, I. S.; Challa, A.; Derickson, B.; Hsia, K. J.; Li, X. Nano Lett. 2010, 10 (10), 3927-3932.

(30) Deneke, C.; Müller, C.; Jin-Phillipp, N.; Schmidt, O. Semicond. Sci. Technol. 2002, 17 (12), 1278.

(31) Huang, M.; Boone, C.; Roberts, M.; Savage, D. E.; Lagally, M. G.; Shaji, N.; Qin, H.; Blick, R.; Nairn, J. A.; Liu, F. Adv. Mater. 2005, 17 (23), 2860-2864.

(32) Cianci, E.; Schina, A.; Minotti, A.; Quaresima, S.; Foglietti, V. Sens. Actuators, A 2006, 127 (1), 80-87.

(33) Tabata, O.; Kawahata, K.; Sugiyama, S.; Igarashi, I. In Mechanical property measurements of thin films using load-deflection of composite rectangular membrane; Micro Electro Mechanical Systems, IEEE Proceedings, An Investigation of Micro Structures, Sensors, Actuators, Machines and Robots; IEEE: Bellingham, WA, 1989; pp $152-156$.

(34) Froeter, P.; Yu, X.; Huang, W.; Du, F.; Li, M.; Chun, I.; Kim, S. H.; Hsia, K. J.; Rogers, J. A.; Li, X. Nanotechnology 2013, 24 (47), 475301.

(35) Prinz, V. Y.; Golod, S. J. Appl. Mech. Tech. Phys. 2006, 47 (6), 867-878. 